\title{
Review Article \\ MicroRNAs Involvement in Radioresistance of Head and Neck Cancer
}

\author{
Parwez Ahmad, ${ }^{1}$ Jiri Sana, ${ }^{1,2}$ Marek Slavik, ${ }^{3}$ Pavel Slampa, ${ }^{3}$ \\ Pavel Smilek, ${ }^{4}$ and Ondrej Slaby ${ }^{1,2}$ \\ ${ }^{1}$ Central European Institute of Technology, Masaryk University, Brno, Czech Republic \\ ${ }^{2}$ Department of Comprehensive Cancer Care, Masaryk Memorial Cancer Institute, Faculty of Medicine, \\ Masaryk University, Brno, Czech Republic \\ ${ }^{3}$ Department of Radiation Oncology, Masaryk Memorial Cancer Institute, Brno, Czech Republic \\ ${ }^{4}$ Department of Otorhinolaryngology and Head and Neck Surgery, St. Anne's Faculty Hospital, \\ Masaryk University, Brno, Czech Republic
}

Correspondence should be addressed to Ondrej Slaby; on.slaby@gmail.com

Received 27 December 2016; Accepted 12 February 2017; Published 23 February 2017

Academic Editor: Alvaro González

Copyright (C) 2017 Parwez Ahmad et al. This is an open access article distributed under the Creative Commons Attribution License, which permits unrestricted use, distribution, and reproduction in any medium, provided the original work is properly cited.

\begin{abstract}
Resistance to the ionizing radiation is a current problem in the treatment and clinical management of various cancers including head and neck cancer. There are several biological and molecular mechanisms described to be responsible for resistance of the tumors to radiotherapy. Among them, the main mechanisms include alterations in intracellular pathways involved in DNA damage and repair, apoptosis, proliferation, and angiogenesis. It has been found that regulation of these complex processes is often controlled by microRNAs. MicroRNAs are short endogenous RNA molecules that posttranscriptionally modulate gene expression and their deregulated expression has been observed in many tumors including head and neck cancer. Specific expression patterns of microRNAs have also been shown to predict prognosis and therapeutic response in head and neck cancer. Therefore, microRNAs present promising biomarkers and therapeutic targets that might overcome resistance to radiation and improve prognosis of head and neck cancer patients. In this review, we summarize the current knowledge of the functional role of microRNAs in radioresistance of cancer with special focus on head and neck cancer.
\end{abstract}

\section{Introduction}

Head and neck cancers are the sixth most common cancers worldwide that are represented mainly by the squamous cell carcinoma (HNSCC) occurring in the oral cavity, pharynx, or larynx. Etiological causes of HNSCC development are excessive use of tobacco and alcohol and infection with human papilloma virus (HPV), especially HPV 16 and HPV 18, and Epstein-Barr virus (EBV) [1,2]. The current standard therapy consists of radical surgical resection followed by adjuvant radiotherapy (RT) in monotherapy or concomitantly with chemotherapy or targeted therapy. However, the biological behavior of HNSCC is unpredictable at this time and there are many patients resistant to the administrated therapy.

In this regard, great efforts are being made for elucidation of radioresistance mechanisms as well as discovery of new prognostic and predictive biomarkers in response to the ionizing radiation (IR) that remains one of the cornerstones of head and neck cancer treatment [2]. IR may affect cellular components directly and/or indirectly by the generation of highly reactive oxygen species, known as free radicals, that react with other molecules in very short time. The major effect of this process in cells is DNA doublestrand breaks (DSB), which ultimately lead to the extensive genome instability and cell death. Nevertheless, there are cellular mechanisms and tumor microenvironment factors that repair and/or prevent these molecular changes, respectively. High activity of these mechanisms supported by tumor microenvironment led to the increased resistance of cancer cells to the IR and, subsequently, to the RT failures, early tumor recurrences, and poor prognoses of cancer patients [3]. 
Many recent studies have showed that very important molecular players closely related to the response to the IR at the cellular level are among other microRNAs (miRNAs). MiRNAs are endogenous, evolutionary conserved, small noncoding RNAs, 18-25 nucleotides in length, which regulate gene expression by binding to $3^{\prime} \mathrm{UTR}$ of targeted mRNA. MiRNAs are involved in the regulation of all important cellular processes associated with response to the IR such as DNA damage response and repair, apoptosis, proliferation, and angiogenesis. Moreover, these molecules have been published to be deregulated in many cancers including HNSCC $[2,4]$ and, thus, miRNAs seem to be key regulators of HNSCC response to the IR. The aim of this review is to summarize present knowledge about radioresistance of HNSCC with emphasis on role of miRNAs in this tumor phenomenon.

\section{Radioresistance in Head and Neck Cancer}

Tumor resistance to the ionizing radiation is a complex process depending on the many biological factors and cellular mechanisms regulated by the intrinsic cell signaling network. The most frequently mentioned events leading to the development of HNSCC radioresistance are disruption of proliferation, apoptosis, DNA damage response and repair, and angiogenic signaling in the tumor [29-31].

\subsection{Alterations in EGFR, PIBK/AKT, and RAS Pathways.} Very important signaling pathway regulating mechanisms mentioned above seem to be EGFR (epidermal growth factor receptor) signaling network triggered canonically by the transmembrane protein with tyrosine kinase activity that is overexpressed in about $90 \%$ of HNSCC. High EGFR expression correlates with poor prognosis of patients and resistance to conventional radiotherapy. Most studies have also demonstrated a lower local control after radiation of tumors overexpressing EGFR. The protein overexpression is thought to result from enhanced transcription, whereas gene amplification has been observed less frequently [30, 32]. Stimulation of this receptor may activate many downstream molecules, such as PI3K (phosphatidylinositol-3-kinase) and RAS. PI3K is able to activate AKT that phosphorylates multiple downstream effectors controlling cell survival and apoptosis as well as DNA damage repair and epithelial to mesenchymal transition. In turn, RAS is able to directly stimulate a tyrosine kinase cascade, which comprises in order B-Raf, MEK, and finally mitogen-activated protein kinase (MAPK). MAPK phosphorylates Myc, FOS, and Jun, allowing their nuclear translocation, which leads to cell proliferation [3].

2.2. Deregulation of TP53 Associated Intrinsic Apoptosis. TP53 is a tumor suppressor gene which is a key regulator of genome stability through the modulation of DNA damage response. About $40-70 \%$ of HNSCC has mutation in TP53 gene, leading to inactivation of its protein product [30, 33]. Alteration in p53 leads to an impaired capability of cell cycle arrest and to inhibition of the apoptosis. As a consequence, tumor cells carrying TP53 mutation are less sensitive to radiation-induced cell death and are unable to restore DNA integrity, thus accumulating several genetic mutations which lead to increased tumor heterogeneity and finally to resistance to conventional radiotherapy $[3,30]$. Moreover, several evidences suggest that it is necessary to distinguish carefully TP53 mutations, which are related to the prognosis in HNSCC. In the other words, prognosis of patients varies depending on the particular mutated TP53 protein domain $[30,34,35]$. One of the main classifications of TP53 mutations divide these to disruptive and not disruptive; any mutation in L2 or L3 loop of the DNA binding domain resulting in a polarity change of the protein or any stop codon was classified as disruptive. Disruptive TP53 mutations were associated with poor outcome and increased radioresistance in HNSCC $[30,36,37]$.

2.3. Hypoxia Induced Neovascularization. Although angiogenesis plays the main role, it is not the only way to develop a vasculature. Another mechanism is called vasculogenesis and is observed mainly in the stage of embryo development where it gives rise to the first primitive vascular plexus from circulating cells. Angiogenesis is then responsible for the remodeling and expansion of this network [38]. Because tumor irradiation abrogates local angiogenesis, the tumor must rely on the vasculogenesis pathway for regrowth after irradiation. Irradiated tumor produces a marked influx of $\mathrm{CD} 11 \mathrm{~b}+$ macrophages into the tumor, and these are crucial to the formation of new blood vessels. This process is driven by increased tumor hypoxia that manifests in stabilization of HIF- $1 \alpha$ and HIF- $1 \beta$ heterodimer (hypoxia-inducible factor 1) and upregulation of SDF-1 (stromal cell-derived factor 1 or CXCL12). Disruption of the above signalization prevents the radiation-induced influx of the $\mathrm{CD} 11 \mathrm{~b}+$ macrophages and delays tumor recurrence [39-41].

HIF1 also induces proangiogenic growth factors such as FGF (fibroblast growth factor) and VEGF (vascular endothelial growth factors) that stimulate endothelial cells to secrete several proteases and plasminogen activators resulting in the degradation of the vessel basement membrane, which in turn allows cells to invade the surrounding matrix. The cells migrate, proliferate, and eventually differentiate to form a new vessel [42]. Finally, VEGF is also able to activate PI3K/AKT and RAS/MAPK signaling pathways and, thus, is involved in the control of cell survival, proliferation, and apoptosis as described above.

2.4. Epithelial-Mesenchymal Transition. EMT (epithelialmesenchymal transition) is a process that allows a polarized epithelial cell, which normally interacts with basement membrane via its basal surface, to undergo multiple biochemical changes that enable it to assume the mesenchymal cell phenotype, which includes enhanced migratory capacity, invasiveness, elevated resistance to apoptosis, and greatly increased production of ECM components [43,44]. A crucial step of EMT is the loss of E-cadherin, a strong epithelial marker involved in adherent junction that anchors epithelial cells to each other. Loss or decrease of E-cadherin expression causes the translocation of $\beta$-catenin protein from the cell membrane to the nucleus to induce transcription of 
EMT-related genes, such as TWIST and SNAIL1. Another important protein involved in EMT is vimentin, which is an intermediate filament protein used as a marker for mesenchymal cells and is associated with the migratory phenotype, local recurrence, and survival in HNSCC $[29,30]$. Emerging evidence suggests that EMT has also a crucial role in cancer radiation resistance primarily via enhancing selfrenewal and other CSCs (cancer stem cells) characteristics [45-47].

2.5. Cancer Stem Cells. Most cancers, including HNSCC, contain a small subpopulation of cells that, like the stem cells, are characterized by the ability of self-renewal, unlimited and slow proliferation, and differentiation potential. Moreover, these cellular populations called cancer stem cells (CSCs) have also unique potential for tumor initiation and are highly resistant to both chemotherapy and ionizing radiation [48]. Conventional therapeutic approaches are successful in debulking the tumor through targeting of highly proliferating cells. However, as outlined above, the slow-growing CSCs evade conventional therapies, and, with the passage of time, these cells are activated and regenerate tumors locally or at distant sites [49]. This is one of possible causes explaining the relatively high recurrence rates in patients with HNSCC. CSCs are located in the invasive fronts of HNSCC close to blood vessels and express markers such as ALDH (aldehyde dehydrogenase), CD133, CD24, and CD44 [50, 51]. In HNSCC patients, high percentage of CD44 positive cells was associated with higher rate of treatment failure in general, while cells expressing CD44, CD24, Oct4, and integrin $\beta 1$ were associated with poor outcome after radiotherapy [52].

\section{Involvement of miRNAs in Radioresistance of Cancer}

It has been suggested that miRNAs can modulate tumor radioresistance by affecting radiation-related signaling pathways which are involved in DNA damage repair, cell cycle regulation, apoptosis, neovascularization, and inflammation. Among these pathways are EGFR, PI3K/AKT, NF-kB, RASMAPK, and TGF- $\beta$, as well as JAK-STAT signaling $[53,54]$. Well described miRNA that regulates response to the ionizing radiation through inhibition of $\mathrm{PI} 3 \mathrm{~K} / \mathrm{AKT}$ pathway is miR21. Interestingly, this process was also accompanied by the enhancement of autophagy [55]. Abnormal expression of, inter alia, miR-21 has been associated also with radioresistance in lung carcinoma stem-like cells [56] and found in high-risk HPV positive cervical cancer cells where this miR21 regulates LATS-1 (large tumor suppressor kinase 1) [57]. Glioma radioresistance is via AKT signalization regulated also by the miR-221/222. In this case, activation of AKT has been independent of PTEN status [58]. Nevertheless, the same miRNAs target PTEN and regulate radioresistance in gastric carcinoma cells [59]. PTEN/PI3K/AKT signaling pathway is further regulated by miR-20a. Activation of this pathway results in induction of radioresistance in hepatocellular carcinoma [60]. On the other hand, increased radiosensitivity of hepatic origin tumors has been described after downregulation of miR-210 [61], whereas the same miRNA is also involved in HIF- $1 \alpha / \mathrm{Bcl}-2$ guided radiosensitivity in colon cancer [62]. The close relationship of miR-210 with hypoxia induced cellular processes has been observed also in cervical and breast cancer [63]. Other miRNAs described in relation to breast carcinoma radioresistance are miR-200c, miR-155, and miR-205. However, the molecular mechanisms of their action are not identical. Whereas miR-200c has been found to enhance radiosensitivity by inhibiting autophagy by targeting UBQLN1 [64], miR-155 regulates DNA repair and enhances radiosensitivity by targeting RAD51 [65]. MiR205 has been published to be downregulated in radioresistant breast cancer cells and targets ZEB1 and Ubc13 [66]. In glioblastoma, miR-128a, miR-135b, miR-1, miR125a, miR-150, and miR-425 were found to be associated with radioresistance [67-69]. Moreover, our recent study suggests that miR-338-5p sensitizes glioblastoma cells to radiation through regulation of genes involved in DNA damage response such as NDFIP1 (Nedd4 Family Interacting Protein 1), RHEB (RAS homolog enriched in brain), and PPP2R5A (Protein Phosphatase 2 Regulatory Subunit B', Alph) [70]. In lung cancer loss of miR-1323 and/or miR-18a enhances radiosensitivity whereas miR-511 has been found to suppress growth of radioresistant cell lines [71-73]. More deep study on molecular mechanisms revealed that effect induced by miR-1323 is mainly through the suppressing of PRKDC (Protein Kinase, DNA-Activated, Catalytic Polypeptide) [71]. Finally, both miR-100 and miR-124 have been found to be associated with radiosensitivity in colorectal carcinoma trough targeting PRRX1 (Paired Related Homeobox 1) $[74,75]$.

\section{Involvement of miRNAs in Radioresistance of Head and Neck Cancer}

Biological mechanisms and signaling network responsible for radioresistance of HNSCC are very similar to other cancers described above. Therefore, it is not surprising that there are growing evidence supporting the role of miRNAs in response to ionizing radiation also in carcinomas of the head and neck. In HNSCC, ATM-mediated radiosensitivity is regulated by downstream targets such as SMC1 and Snail. In absence of ATM function, miR-16, miR-29b, miR-1254, and miR-150 were found to be upregulated, while let-7e was downregulated [8]. Interestingly, overexpression of miR-150 in glioblastoma promotes radioresistance through upregulation of the cell cycle checkpoint response and its direct targeting led to the higher sensitivity of glioblastoma cells to irradiation [69]. Another study has described that miR-196a promotes an oncogenic effect in HNSCC by suppressing ANXA1 and thus enhancing radioresistance [9]. Similarly, miR-210 is associated with radioresistance in HNSCC, whereas its higher expression has been found in hypoxic cells [10]. Li et al. published a comprehensive study focused on miRNAs in nasopharyngeal carcinoma (NPC). Using next-generation sequencing technology, they revealed miR-37la-5p, miR-34c$5 \mathrm{p}$, and miR-1323 to be overexpressed while miR-324-3p, miR-93-3p, and miR-4501 were downregulated in radioresistant NPC cells [11]. Oncogenic feature of miR-1323 was supported also by Li et al. who published that knockdown 
of miR-1323 restores sensitivity to radiation in radiationresistant lung cancer cells [71], whereas miR-324-3p has been found downregulated in radioresistant NPC cells and directly targets WNT2B gene [21]. On the other hand, miR$34 \mathrm{a}$, another member of the tumor suppressive miR-34 family, shows rather opposite effect on radioresistance than described in Li et al's study. Hypofractionated radiotherapy induced miR-34a expression and enhanced apoptosis in NPC cells [22]. In these cells, miR-205 downregulation determined radioresistance by targeting of PTEN [12], miR-451 increases radiosensitivity by targeting RAB14 (RAS related protein 14) [5], miR-23a is associated with modulation of radioresistance by targeting IL8 [13], and miR-185-3p regulates radioresistance, similar to miR-324-3p, by targeting WNT2B [23]. Low expression of miR-203 has been found to be correlated with local disease recurrence after radiotherapy in a series of patients with laryngeal cancer [27] whereas, in NPC, miR-203 plays important role in radiosensitivity by targeting IL8/AKT [28]. On the other hand, downregulation of miR-21 enhances radiosensitivity, but the responsible mechanism has to be clarified in NPC. MiR-21 is deeply characterized in ESCC, where its overexpression is related to decrease of PTEN expression in radioresistant cell lines [16, 17]. In ESCC, the sensitivity to the radiation is regulated also by miR-381 and miR-96. Expression levels of miR-381 positively correlate with good response of cells in vitro [24], miR-96 shows opposite effect, and its upregulation led to loss of radiosensitivity [14].

In LSCC (laryngeal squamous cell carcinoma), upregulation of miR-296-5p regulates radiosensitivity by targeting MDR1 gene [15]. Upregulation of miR-24 has been found to be associated with radiosensitivity through targeting of XIAP (X-linked inhibitor of apoptosis protein) in LSCC and NPC $[19,20]$. In OSCC (oral squamous cell carcinoma), miR$125 \mathrm{~b}$ regulates proliferation and radiosensitivity by targeting ICAM2 [25] and miR-17-5p regulates expression of p21 [26]. At the end, upregulation of miR-31 enhances radiosensitivity in esophageal adenocarcinoma cell lines probably by activating HIF1 [6,7]. An involvement of miRNAs in the radioresistance of head and neck cancer is summarized in Table 1 and Figure 1.

\section{Conclusion}

Head and neck cancers account for about $3 \%$ of all cancers in developed countries and their etiology is associated with tobacco, alcohol, and sexually transmitted infection of human papilloma virus and Epstein-Barr virus. Therefore, it is possible to assume that the incidence will rise in the future. The biological behavior and prognosis of head and neck cancer is unpredictable at this time, radiotherapy often fails, and early recurrences are observed. This is primarily caused by the resistance of tumors to radiotherapy. Overcoming the resistance is nowadays one of the main challenges in head and neck cancers research. Recently, several signaling pathways have been described to be associated with resistance of head and neck cancer cells to ionizing radiation. Many genes involved in these pathways are regulated by miRNAs,
TABLE 1: Expression of radioresistance associated miRNAs in head and neck cancers.

\begin{tabular}{|c|c|c|c|}
\hline $\begin{array}{l}\text { Expression in } \\
\text { tumor }\end{array}$ & miRNA & Carcinoma type & Reference \\
\hline \multirow{16}{*}{ Upregulated } & miR-451 & NPC & [5] \\
\hline & miR-31 & $\mathrm{OAC}$ & {$[6,7]$} \\
\hline & miR-150 & HNSCC & {$[8]$} \\
\hline & miR-1254 & HNSCC & [8] \\
\hline & miR-16 & HNSCC & [8] \\
\hline & miR-29b & HNSCC & {$[8]$} \\
\hline & miR-196a & HNSCC & [9] \\
\hline & miR-210 & HNSCC & {$[10]$} \\
\hline & miR-1323 & NPC & {$[11]$} \\
\hline & miR-34c-5p & NPC & [11] \\
\hline & miR-371a-5p & NPC & {$[11]$} \\
\hline & miR-205 & NPC & {$[12]$} \\
\hline & miR-23a & NPC & {$[13]$} \\
\hline & miR-96 & ESCC & {$[14]$} \\
\hline & miR-296-5p & LSCC & [15] \\
\hline & miR-21 & NPC & {$[16,17]$} \\
\hline \multirow{15}{*}{ Downregulated } & miR-324-3p & NPC & {$[11]$} \\
\hline & miR-141 & ESCC & {$[18]$} \\
\hline & miR-18b & ESCC & {$[18]$} \\
\hline & miR-301a & ESCC & {$[18]$} \\
\hline & miR-24 & LSCC, NPC & {$[19,20]$} \\
\hline & miR-let 7e & HNSCC & [8] \\
\hline & miR-4501 & NPC & [11] \\
\hline & miR-93-3p & NPC & {$[11]$} \\
\hline & miR-324-3p & NPC & [21] \\
\hline & miR-34a & NPC & {$[22]$} \\
\hline & miR-185-3p & NPC & {$[23]$} \\
\hline & miR-381 & ESCC & {$[24]$} \\
\hline & miR-125b & OSCC & {$[25]$} \\
\hline & miR-17-5p & OsCC & [26] \\
\hline & miR-203 & LSCC, NPC & {$[27,28]$} \\
\hline
\end{tabular}

HNSCC: head and neck squamous cell carcinoma; NPC: nasopharyngeal carcinoma; OSCC: oral squamous cell carcinoma; OAC: oral adenocarcinoma; LSCC: laryngeal squamous cell carcinoma; ESCC: esophageal squamous cell carcinoma.

and direct deregulation of many miRNAs was shown to affect sensitivity to radiation in cellular models. Thus, miRNAs are promising both prognostic and predictive markers, as well as therapeutic targets, which may enable overcoming the resistance of head and neck cancers to the radiotherapy and improve therapeutic results in patients afflicted with these malignancies.

\section{Competing Interests}

The authors declare that they have no competing interests. 


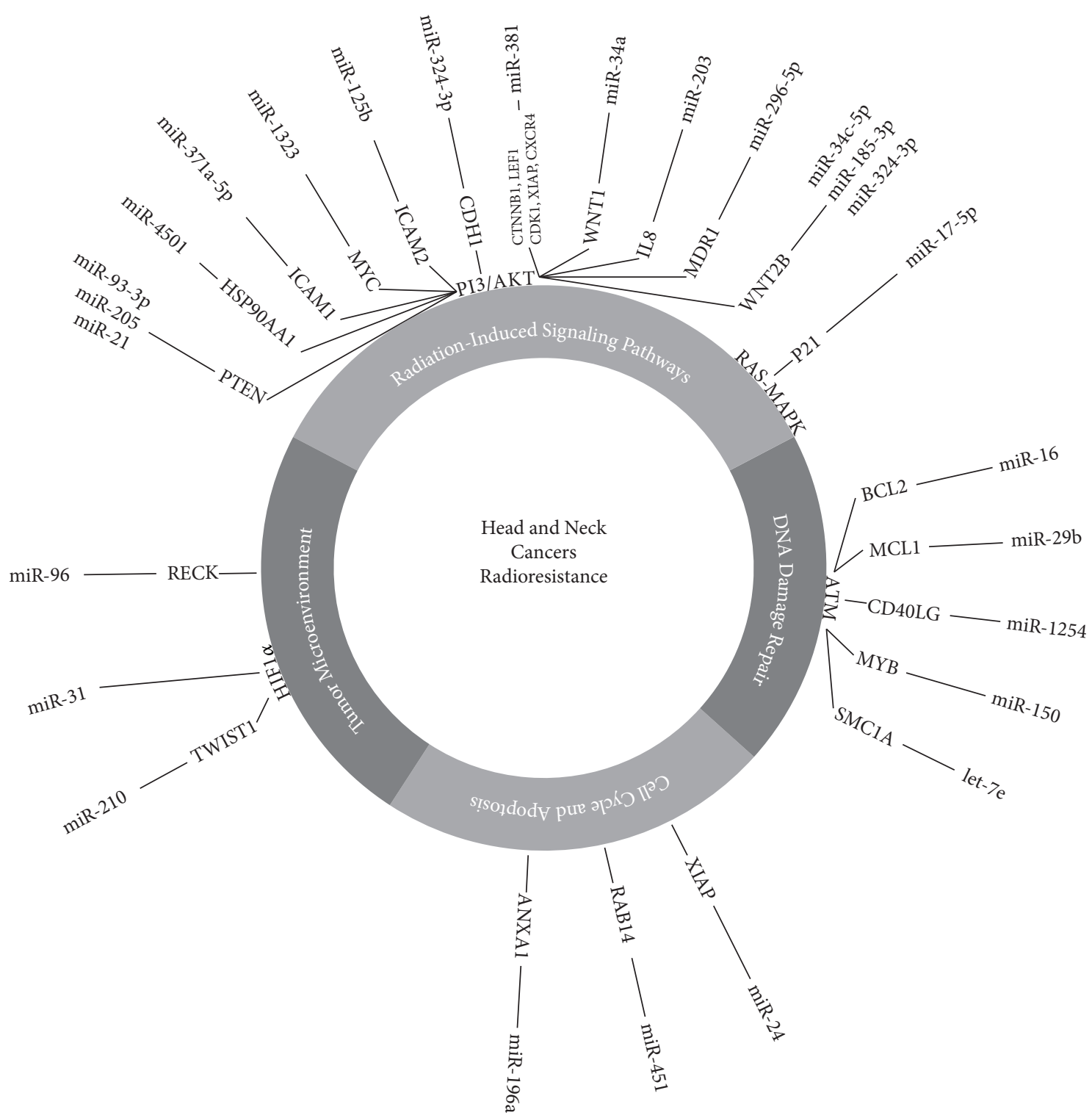

Figure 1: MiRNAs involved in radioresistance of head and neck cancer. This figure shows a regulation of key processes involved in head and neck cancers radioresistance through miRNAs and their targets. ANXA1: Annexin 1, BCL2: B-cell CLL/Lymphoma 2, ATM: Ataxia Telangiectasia Mutated, MCL1: Myeloid cell Leukemia 1, CD40LG: CD40 Ligand, MYB v-Myb: avian myeloblastosis viral oncogene homolog, SMC1A: Structural Maintenance of Chromosomal 1 A, CDH 1: Cadherin 1, PTEN: Phosphatase and Tensin homolog, XIAP: X-linked Inhibitor of Apoptosis E3 ubiquitin Protein, WNT2B: wingless-type MMTV integration site family member 2B, RAB14: member of RAS oncogene family, HIF-1 $\alpha$ : hypoxia-inducible factor 1 alpha subunit, TGF-WNT: transforming growth factor-wingless-type MMTV integration site family, RECK: reversion-inducing cysteine rich protein with Kazal motifs, MDR1: Multi-Drug Resistance gene 1, IL8: Interleukin 8, HSP90AA1: Heat Shock protein 90 kDa Alpha (cytosolic) Class A Member 1, ICAM 1: Intercellular Adhesion Molecule 1, Myc v-Myc: Avian Myelocytomatosis viral oncogene homolog, ICAM 2: Intercellular Adhesion Molecule 2, CTNNB1: Catenin (Cadherin associated protein) Betal, LEF1: Lymphoid Enhancer-Binding Factor 1, CDK1: Cyclin-Dependent Kinase 1, CXCR4: Chemokine (c-X-c motif) Receptor 4, WNT1: wingless-type MMTV integration site family member 1, TWIST1: Twist Basic Helix-Loop-Helix Transcription Factor 1.

\section{Authors' Contributions}

Parwez Ahmad and Jiri Sana contributed equally to this work.

\section{Acknowledgments}

This work was financially supported by the Czech Ministry of Health, Grant no. 15-31627A, Project MZ CR - RVO (MOU,
00209805), and by the Ministry of Education, Youth and Sports of the Czech Republic under Project CEITEC 2020 (LQ1601).

\section{References}

[1] J. Janiszewska, M. Szaumkessel, and K. Szyfter, "MicroRNAs are important players in head and neck carcinoma: a review," 
Critical Reviews in Oncology/Hematology, vol. 88, no. 3, pp. 716728, 2013.

[2] F. Cellini, A. G. Morganti, D. Genovesi, N. Silvestris, and V. Valentini, "Role of microRNA in response to ionizing radiations: evidences and potential impact on clinical practice for radiotherapy," Molecules, vol. 19, no. 4, pp. 5379-5401, 2014.

[3] F. Perri, R. Pacelli, G. Della Vittoria Scarpati et al., "Radioresistance in head and neck squamous cell carcinoma: biological bases and therapeutic implications," Head and Neck, vol. 37, no. 5, pp. 763-770, 2015.

[4] G. Courthod, P. Franco, L. Palermo, S. Pisconti, and G. Numico, "The role of microRNA in head and neck cancer: current knowledge and perspectives," Molecules, vol. 19, no. 5, pp. 57045716, 2014.

[5] T. Zhang, Q. Sun, T. Liu et al., "MiR-451 increases radiosensitivity of nasopharyngeal carcinoma cells by targeting ras-related protein 14 (RAB14)," Tumor Biology, vol. 35, no. 12, pp. 1259312599, 2014.

[6] N. Lynam-Lennon, J. V. Reynolds, L. Marignol, O. M. Sheils, G. P. Pidgeon, and S. G. Maher, "MicroRNA-31 modulates tumour sensitivity to radiation in oesophageal adenocarcinoma," Journal of Molecular Medicine, vol. 90, no. 12, pp. 1449-1458, 2012.

[7] C.-J. Liu, M.-M. Tsai, P.-S. Hung et al., "miR-31 ablates expression of the HIF regulatory factor FIH to activate the HIF pathway in head and neck carcinoma," Cancer Research, vol. 70, no. 4, pp. 1635-1644, 2010.

[8] N. Liu, R. J. Boohaker, C. Jiang, J. R. Boohaker, and B. Xu, "A radiosensitivity MiRNA signature validated by the TCGA database for head and neck squamous cell carcinomas," Oncotarget, vol. 6, no. 33, pp. 34649-34657, 2015.

[9] Y.-E. Suh, N. Raulf, J. Gäken et al., "MicroRNA-196a promotes an oncogenic effect in head and neck cancer cells by suppressing annexin Al and enhancing radioresistance," International Journal of Cancer, vol. 137, no. 5, pp. 1021-1034, 2015.

[10] H. E. Gee, C. Camps, F. M. Buffa et al., "hsa-mir-210 is a marker of tumor hypoxia and a prognostic factor in head and neck cancer," Cancer, vol. 116, no. 9, pp. 2148-2158, 2010.

[11] G. Li, Y. Qiu, Z. Su et al., "Genome-wide analyses of radioresistance-associated miRNA expression profile in nasopharyngeal carcinoma using next generation deep sequencing," PLoS ONE, vol. 8, no. 12, Article ID e84486, 2013.

[12] C. Qu, Z. Liang, J. Huang et al., "MiR-205 determines the radioresistance of human nasopharyngeal carcinoma by directly targeting PTEN," Cell Cycle, vol. 11, no. 4, pp. 785-796, 2012.

[13] X.-H. Li, J.-Q. Qu, H. Yi et al., "Integrated analysis of differential miRNA and mRNA expression profiles in human radioresistant and radiosensitive nasopharyngeal carcinoma cells," PLoS ONE, vol. 9, no. 1, Article ID e87767, 2014.

[14] H. Xia, S. Chen, K. Chen, H. Huang, and H. Ma, "MiR96 promotes proliferation and chemo- or radioresistance by down-regulating RECK in esophageal cancer," Biomedicine and Pharmacotherapy, vol. 68, no. 8, pp. 951-958, 2014.

[15] D. Maia, A. C. de Carvalho, M. A. Horst, A. L. Carvalho, C. Scapulatempo-Neto, and A. L. Vettore, "Expression of miR-296$5 p$ as predictive marker for radiotherapy resistance in earlystage laryngeal carcinoma," Journal of Translational Medicine, vol. 13, article 262, 2015.

[16] H. Zhu, X. Zhu, G. Cheng, M. Zhou, and W. Lou, "Downregulation of microRNA-21 enhances radiosensitivity in nasopharyngeal carcinoma," Experimental and Therapeutic Medicine, vol. 9, no. 6, pp. 2185-2189, 2015.
[17] S. Huang, X.-Q. Li, X. Chen, S.-M. Che, W. Chen, and X.-Z. Zhang, "Inhibition of microRNA-21 increases radiosensitivity of esophageal cancer cells through phosphatase and tensin homolog deleted on chromosome 10 activation," Diseases of the Esophagus, vol. 26, no. 8, pp. 823-831, 2013.

[18] H. Su, X. Jin, X. Zhang et al., "Identification of microRNAs involved in the radioresistance of esophageal cancer cells," Cell Biology International, vol. 38, no. 3, pp. 318-325, 2014.

[19] L. Xu, Z. Chen, F. Xue et al., "MicroRNA-24 inhibits growth, induces apoptosis, and reverses radioresistance in laryngeal squamous cell carcinoma by targeting X-linked inhibitor of apoptosis protein," Cancer Cell International, vol. 15, article 61, 2015.

[20] S. Wang, R. Zhang, F. X. Claret, and H. Yang, "Involvement of microRNA-24 and DNA methylation in resistance of nasopharyngeal carcinoma to ionizing radiation," Molecular Cancer Therapeutics, vol. 13, no. 12, pp. 3163-3174, 2014.

[21] G. Li, Y. Liu, Z. Su et al., "MicroRNA-324-3p regulates nasopharyngeal carcinoma radioresistance by directly targeting WNT2B," European Journal of Cancer, vol. 49, no. 11, pp. 25962607, 2013.

[22] Z. Long, B. Wang, D. Tao, Y. Huang, and Z. Tao, "Hypofractionated radiotherapy induces miR-34a expression and enhances apoptosis in human nasopharyngeal carcinoma cells," International Journal of Molecular Medicine, vol. 34, no. 5, pp. 13881394, 2014.

[23] G. Li, Y. Wang, Y. Liu et al., "miR-185-3p regulates nasopharyngeal carcinoma radioresistance by targeting WNT2B in vitro," Cancer Science, vol. 105, no. 12, pp. 1560-1568, 2014.

[24] S. Zhou, W. Ye, J. Ren et al., "MicroRNA-381 increases radiosensitivity in esophageal squamous cell carcinoma," American Journal of Cancer Research, vol. 5, pp. 267-277, 2015.

[25] M. Shiiba, K. Shinozuka, K. Saito et al., "MicroRNA-125b regulates proliferation and radioresistance of oral squamous cell carcinoma," British Journal of Cancer, vol. 108, no. 9, pp. 18171821, 2013.

[26] S.-Y. Wu, K.-C. Lin, J.-F. Chiou et al., "MicroRNA-17-5p posttranscriptionally regulates p21 expression in irradiated betel quid chewing-related oral squamous cell carcinoma cells," Strahlentherapie und Onkologie, vol. 189, no. 8, pp. 675-683, 2013.

[27] M. C. de Jong, J. J. Ten Hoeve, R. Grénman et al., "Pretreatment microRNA expression impacting on epithelial-tomesenchymal transition predicts intrinsic radiosensitivity in head and neck cancer cell lines and patients," Clinical Cancer Research, vol. 21, no. 24, pp. 5630-5638, 2015.

[28] J.-Q. Qu, H.-M. Yi, X. Ye et al., "MiRNA-203 reduces nasopharyngeal carcinoma radioresistance by targeting IL8/AKT signaling," Molecular Cancer Therapeutics, vol. 14, no. 11, pp. 26532664, 2015.

[29] C. Metheetrairut and F. J. Slack, "MicroRNAs in the ionizing radiation response and in radiotherapy," Current Opinion in Genetics and Development, vol. 23, no. 1, pp. 12-19, 2013.

[30] F. Ganci, A. Sacconi, V. Manciocco et al., "Radioresistance in head and neck squamous cell carcinoma-possible molecular markers for local recurrence and new putative therapeutic strategies," in Contemporary Issues in Head and Neck Cancer Management, InTech, 2015.

[31] E. Korpela, D. Vesprini, and S. K. Liu, "MicroRNA in radiotherapy: miRage or miRador?" British Journal of Cancer, vol. 112, no. 5, pp. 777-782, 2015. 
[32] M. Zimmermann, A. Zouhair, D. Azria, and M. Ozsahin, "The epidermal growth factor receptor (EGFR) in head and neck cancer: its role and treatment implications," Radiation Oncology, vol. 1, no. 1, article 11, 2006.

[33] M. Olivier, M. Hollstein, and P. Hainaut, "TP53 mutations in human cancers: origins, consequences, and clinical use," Cold Spring Harbor Perspectives in Biology, vol. 2, no. 1, Article ID a001008, 2010.

[34] A. M. Gross, R. K. Orosco, J. P. Shen et al., "Multi-tiered genomic analysis of head and neck cancer ties TP53 mutation to 3p loss," Nature Genetics, vol. 46, no. 9, pp. 939-943, 2014.

[35] J. K. Peltonen, K. H. Vähäkangas, H. M. Helppi, R. Bloigu, P. Pääkkö, and T. Turpeenniemi-Hujanen, "Specific TP53 mutations predict aggressive phenotype in head and neck squamous cell carcinoma: a retrospective archival study," Head and Neck Oncology, vol. 3, no. 1, article 20, 2011.

[36] M. L. Poeta, J. Manola, M. A. Goldwasser et al., "TP53 mutations and survival in squamous-cell carcinoma of the head and neck," The New England Journal of Medicine, vol. 357, no. 25, pp. 25522561, 2007.

[37] H. D. Skinner, V. C. Sandulache, T. J. Ow et al., “TP53 disruptive mutations lead to head and neck cancer treatment failure through inhibition of radiation-induced senescence," Clinical Cancer Research, vol. 18, no. 1, pp. 290-300, 2012.

[38] S. Patan, "Vasculogenesis and angiogenesis," Cancer Treatment and Research, vol. 117, pp. 3-32, 2004.

[39] H. E. Barker, J. T. E. Paget, A. A. Khan, and K. J. Harrington, "The tumour microenvironment after radiotherapy: mechanisms of resistance and recurrence," Nature Reviews Cancer, vol. 15, no. 7, pp. 409-425, 2015.

[40] T. W. H. Meijer, J. H. A. M. Kaanders, P. N. Span, and J. Bussink, "Targeting hypoxia, HIF-1, and tumor glucose metabolism to improve radiotherapy efficacy," Clinical Cancer Research, vol. 18, no. 20, pp. 5585-5594, 2012.

[41] J. M. Brown, "Vasculogenesis: a crucial player in the resistance of solid tumours to radiotherapy," The British journal of radiology, vol. 87, no. 1035, 2014.

[42] M. J. Cross and L. Claesson-Welsh, "FGF and VEGF function in angiogenesis: signalling pathways, biological responses and therapeutic inhibition," Trends in Pharmacological Sciences, vol. 22, no. 4, pp. 201-207, 2001.

[43] R. Kalluri and E. G. Neilson, "Epithelial-mesenchymal transition and its implications for fibrosis," The Journal of Clinical Investigation, vol. 112, no. 12, pp. 1776-1784, 2003.

[44] R. Kalluri and R. A. Weinberg, "The basics of epithelialmesenchymal transition," Journal of Clinical Investigation, vol. 119, no. 6, pp. 1420-1428, 2009.

[45] L. Chang, P. H. Graham, J. Hao et al., "Acquisition of epithelialmesenchymal transition and cancer stem cell phenotypes is associated with activation of the PI3K/Akt/mTOR pathway in prostate cancer radioresistance," Cell Death and Disease, vol. 4, no. 10, article e875, 2013.

[46] Y.-C. Zhou, J.-Y. Liu, J. Li et al., "Ionizing radiation promotes migration and invasion of cancer cells through transforming growth factor-beta-mediated epithelial-mesenchymal transition," International Journal of Radiation Oncology Biology Physics, vol. 81, no. 5, pp. 1530-1537, 2011.

[47] S. A. Mani, W. Guo, M.-J. Liao et al., "The epithelialmesenchymal transition generates cells with properties of stem cells," Cell, vol. 133, no. 4, pp. 704-715, 2008.

[48] A. Kreso and J. E. Dick, "Evolution of the cancer stem cell model," Cell Stem Cell, vol. 14, no. 3, pp. 275-291, 2014.
[49] R. Kleinová, O. Slabý, and J. Šáňa, “The relevance of microRNAs in glioblastoma stem cells," Klinicka onkologie, vol. 28, no. 5, pp. 338-344, 2015.

[50] S. Krishnamurthy and J. E. Nör, "Head and neck cancer stem cells," Journal of Dental Research, vol. 91, no. 4, pp. 334-340, 2012.

[51] J. Han, T. Fujisawa, S. R. Husain, and R. K. Puri, "Identification and characterization of cancer stem cells in human head and neck squamous cell carcinoma," BMC Cancer, vol. 14, no. 1, article 173, 2014.

[52] M. I. Koukourakis, A. Giatromanolaki, V. Tsakmaki, V. Danielidis, and E. Sivridis, "Cancer stem cell phenotype relates to radio-chemotherapy outcome in locally advanced squamous cell head-neck cancer," British Journal of Cancer, vol. 106, no. 5, pp. 846-853, 2012.

[53] A. Besse, J. Sana, P. Fadrus, and O. Slaby, "MicroRNAs involved in chemo- and radioresistance of high-grade gliomas," Tumor Biology, vol. 34, no. 4, pp. 1969-1978, 2013.

[54] L. Zhao, A. M. Bode, Y. Cao, and Z. Dong, "Regulatory mechanisms and clinical perspectives of miRNA in tumor radiosensitivity," Carcinogenesis, vol. 33, no. 11, pp. 2220-2227, 2012.

[55] H.-S. Gwak, T. H. Kim, G. H. Jo et al., "Silencing of microRNA21 confers radio-sensitivity through inhibition of the PI3K/AKT pathway and enhancing autophagy in malignant glioma cell lines," PLoS ONE, vol. 7, no. 10, Article ID e47449, 2012.

[56] J. Zhang, C. Zhang, L. Hu et al., "Abnormal expression of miR-21 and miR-95 in cancer stem-like cells is associated with radioresistance of lung cancer," Cancer Investigation, vol. 33, no. 5, pp. 165-171, 2015.

[57] S. Liu, L. Song, L. Zhang, S. Zeng, and F. Gao, "MiR-21 modulates resistance of HR-HPV positive cervical cancer cells to radiation through targeting LATS1," Biochemical and Biophysical Research Communications, vol. 459, no. 4, pp. 679-685, 2015.

[58] W. Li, F. Guo, P. Wang, S. Hong, and C. Zhang, "miR-221/222 confers radioresistance in glioblastoma cells through activating Akt independent of PTEN status," Current Molecular Medicine, vol. 14, no. 1, pp. 185-195, 2014.

[59] Z. Chun-Zhi, H. Lei, Z. An-Ling et al., "MicroRNA-221 and microRNA-222 regulate gastric carcinoma cell proliferation and radioresistance by targeting PTEN," BMC Cancer, vol. 10, article $367,2010$.

[60] Y. Zhang, L. Zheng, Y. Ding et al., "MIR-20a Induces Cell Radioresistance by Activating the PTEN/PI3K/Akt Signaling Pathway in Hepatocellular Carcinoma," International Journal of Radiation Oncology Biology Physics, vol. 92, no. 5, pp. 1132-1140, 2015.

[61] W. Yang, T. Sun, J. Cao, F. Liu, Y. Tian, and W. Zhu, "Downregulation of miR-210 expression inhibits proliferation, induces apoptosis and enhances radiosensitivity in hypoxic human hepatoma cells in vitro," Experimental Cell Research, vol. 318, no. 8, pp. 944-954, 2012.

[62] Y. Sun, X. Xing, Q. Liu et al., "Hypoxia-induced autophagy reduces radiosensitivity by the HIF- $1 \alpha / \mathrm{miR}-210 / \mathrm{Bcl}-2$ pathway in colon cancer cells," International Journal of Oncology, vol. 46, no. 2, pp. 750-756, 2015.

[63] M. E. Crosby, R. Kulshreshtha, M. Ivan, and P. M. Glazer, "MicroRNA regulation of DNA repair gene expression in hypoxic stress," Cancer Research, vol. 69, no. 3, pp. 1221-1229, 2009. 
[64] Q. Sun, T. Liu, Y. Yuan et al., "MiR-200c inhibits autophagy and enhances radiosensitivity in breast cancer cells by targeting UBQLN1," International Journal of Cancer, vol. 136, no. 5, pp. 1003-1012, 2015.

[65] P. Gasparini, F. Lovat, M. Fassan et al., "Protective role of miR-155 in breast cancer through RAD51 targeting impairs homologous recombination after irradiation," Proceedings of the National Academy of Sciences of the United States of America, vol. 111, no. 12, pp. 4536-4541, 2014.

[66] P. Zhang, L. Wang, C. Rodriguez-Aguayo et al., "MiR-205 acts as a tumour radiosensitizer by targeting ZEB1 and Ubc13," Nature Communications, vol. 5, article 5671, 2014.

[67] L. Ye, G. Yu, C. Wang et al., "MicroRNA-128a, BMI1 polycomb ring finger oncogene, and reactive oxygen species inhibit the growth of U-87 MG glioblastoma cells following exposure to X-ray radiation," Molecular Medicine Reports, vol. 12, no. 4, pp. 6247-6254, 2015.

[68] S. Xiao, Z. Yang, R. Lv et al., "miR-135b contributes to the radioresistance by targeting GSK $3 \beta$ in human glioblastoma multiforme cells," PLoS ONE, vol. 9, no. 9, Article ID e108810, 2014.

[69] P. Moskwa, P. O. Zinn, Y. E. Choi et al., "A functional screen identifies miRs that induce radioresistance in glioblastomas," Molecular Cancer Research, vol. 12, no. 12, pp. 1767-1778, 2014.

[70] A. Besse, J. Sana, R. Lakomy et al., "MiR-338-5p sensitizes glioblastoma cells to radiation through regulation of genes involved in DNA damage response," Tumor Biology, vol. 37, no. 6, pp. 7719-7727, 2016.

[71] Y. Li, W. Han, T.-T. Ni et al., "Knockdown of microRNA-1323 restores sensitivity to radiation by suppression of PRKDC activity in radiation-resistant lung cancer cells," Oncology Reports, vol. 33, no. 6, pp. 2821-2828, 2015.

[72] H.-H. Zhang, M. Pang, W. Dong et al., "MiR-511 induces the apoptosis of radioresistant lung adenocarcinoma cells by triggering BAX," Oncology Reports, vol. 31, no. 3, pp. 1473-1479, 2014.

[73] Z. Shen, X. Wu, Z. Wang, B. Li, and X. Zhu, "Effect of miR18a overexpression on the radiosensitivity of non-small cell lung cancer," International Journal of Clinical and Experimental Pathology, vol. 8, no. 1, pp. 643-648, 2015.

[74] X.-D. Yang, X.-H. Xu, S.-Y. Zhang et al., "Role of miR-100 in the radioresistance of colorectal cancer cells," American Journal of Cancer Research, vol. 5, pp. 545-559, 2015.

[75] Y. Zhang, L. Zheng, J. Huang et al., "MiR-124 radiosensitizes human colorectal cancer cells by targeting PRRX1," PLoS ONE, vol. 9, no. 4, Article ID e93917, 2014. 


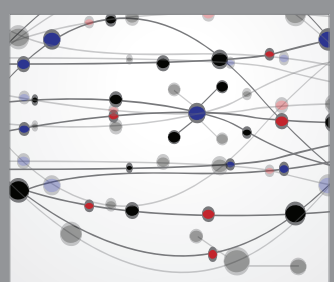

The Scientific World Journal
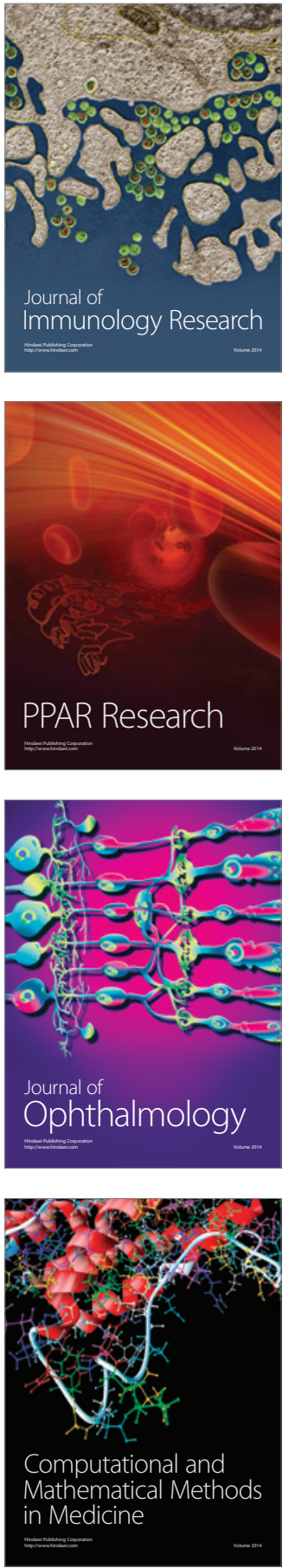

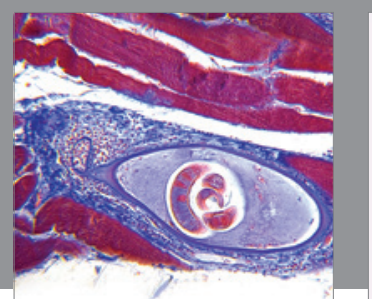

Gastroenterology Research and Practice
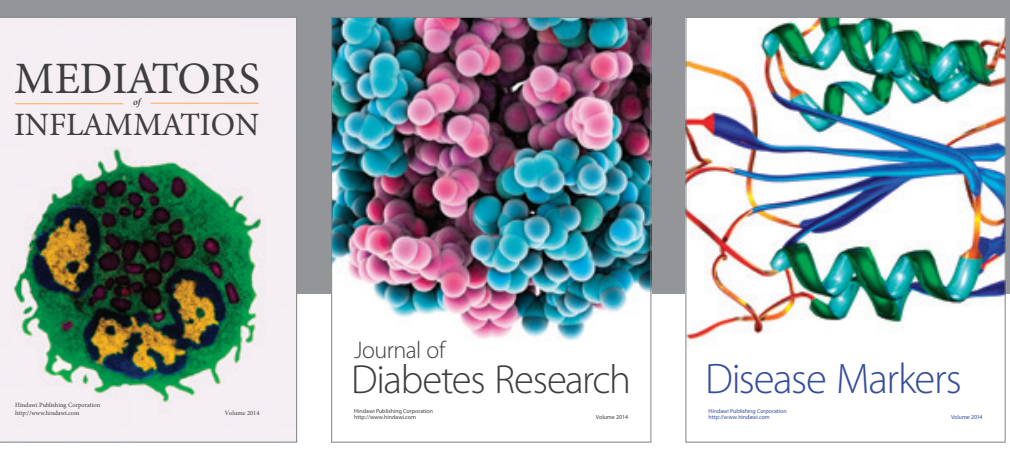

Disease Markers

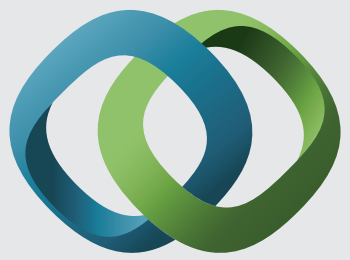

\section{Hindawi}

Submit your manuscripts at

https://www.hindawi.com
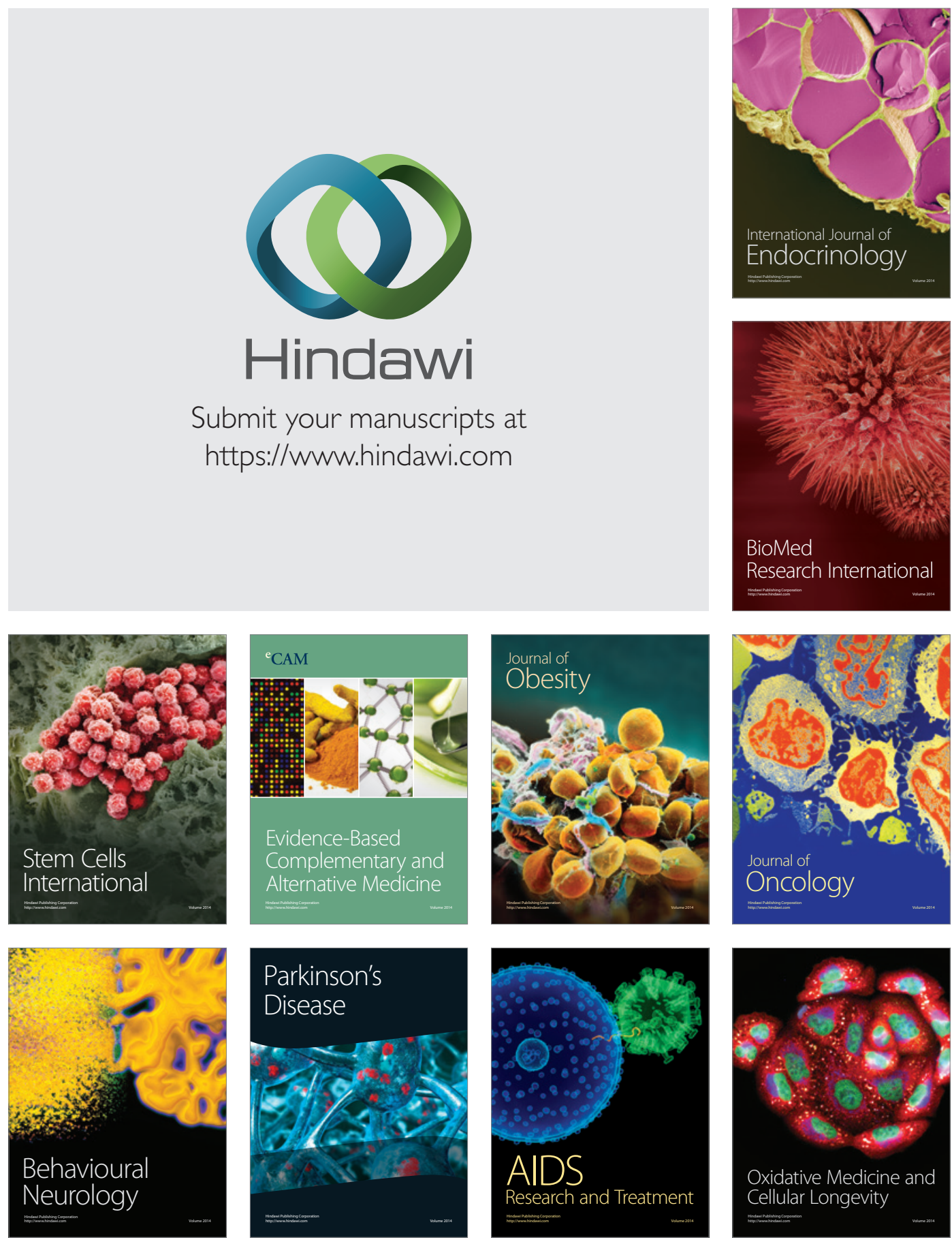